\title{
Le point de vue des fédérations françaises de parents d'élèves
}

Georges Dupon-Lahitte, Christian Janet et Eric Raffin

\section{(2) OpenEdition \\ 1 Journals}

Édition électronique

URL : https://journals.openedition.org/ries/1905

DOI : $10.4000 /$ ries. 1905

ISSN : 2261-4265

Éditeur

France Education international

\section{Édition imprimée}

Date de publication : 1 décembre 2002

Pagination : 65-69

ISBN : 978-2854-2-0555-8

ISSN : $1254-4590$

\section{Référence électronique}

Georges Dupon-Lahitte, Christian Janet et Eric Raffin, « Le point de vue des fédérations françaises de parents d'élèves », Revue internationale d'éducation de Sèvres [En ligne], 31 | décembre 2002, mis en ligne le 24 novembre 2011, consulté le 07 juillet 2021. URL : http://journals.openedition.org/ries/1905 ; DOI : https://doi.org/10.4000/ries.1905 


\title{
Le point de vue des fédérations françaises de parents d'élèves
}

\author{
Georges Dupon-Lahitte, FCPE \\ Christian Janet, PEEP \\ Eric Raffin, UNAPEL
}

\section{Pour les enfants, UNE ÉCOLE AVEC LES PARENTS}

Le renforcement des relations parents/école apparaît à beaucoup comme une nécessité à la fois pour fournir aux élèves un cadre structurant, favorable à leur réussite et leur épanouissement, mieux adapté à la lutte contre la violence, la drogue, mais aussi pour aider l'école à évoluer dans son organisation et ses méthodes afin d'offrir à tous les enfants les atouts indispensables à leur future insertion professionnelle et sociale.

Beaucoup de parents rencontrent au sein des établissements ou des instances académiques une réelle volonté de travailler ensemble dans l'intérêt des enfants. Mais le dialogue n'est pas partout aussi facile.

Malgré la loi d'orientation de 1989 qui fait des parents des partenaires à part entière du système éducatif, ceux-ci sont parfois accusés d'être «trop présents» lorsqu'ils s'avisent de sortir de leur rôle de bailleurs de fonds ou d'organisateurs de kermesses dans lequel on souhaiterait qu'ils se cantonnent. Certains, au sein de l'Éducation nationale, acceptent mal l'intrusion des parents dans un domaine qui leur semble réservé: l'École. A l'inverse, lorsque les parents, pour des raisons diverses, ne fréquentent pas l'établissement de leurs enfants, ils sont souvent trop rapidement présentés comme "démissionnaires» et sont des boucs émissaires tout trouvés pour justifier à bon compte les défaillances et les carences de l'École.

Les voies peuvent être diverses pour renouer le fil d'un dialogue qui paraît aujourd'hui bien difficile à une grande partie des parents, et leur permettre de retrouver le chemin de l'École: formation des enseignants aux relations avec les parents comme l'a initié l'IUFM de Créteil, amélioration de l'information fournie aux familles, amélioration des conditions d'accueil des parents au sein des établissements par une meilleure prise en compte des contraintes parentales et la création de lieux d'écoute et d'échange, reconnaissance effective du rôle de médiation que jouent les parents délégués, 
N'est-ce pas en contribuant à tisser des relations de respect et de confiance réciproque entre les uns et les autres, en favorisant le partage des expériences et des savoirs, que l'on pourra, ensemble, améliorer ce qui doit l'être au sein du système éducatif?

\section{Au RISQUe de SE PERDRE}

Tandis que notre société vit dans la peur de ses enfants, et cherche remède à ses craintes en interpellant tantôt la prison, tantôt l'école, la lancinante question des relations entre celle-ci et les parents semble rester sans réponse véritable. Les acteurs du monde éducatif seraient-ils atteints de surdité chronique, au point de ne pas entendre les messages qu'ils ne cessent cependant de s'adresser? Et comment est-il possible qu'incompréhension et hostilité caractérisent parfois une relation entre des personnes dont l'idéal commun est une mission d'amour, de paix et d'avenir : éduquer un enfant?

La réponse à cette question tient sans doute en ces deux propositions : l'inconnu engendre la crainte; les parents sont l'école.

La première d'entre elles ne suscite sans doute pas un débat très abondant : chacun, qu'il soit enseignant ou parent, comprend aisément qu'en acceptant d'entendre un point de vue différent du sien, il craint de se perdre, d'affaiblir son autorité, de partager son savoir sans certitude de retour, mais il réalise aussi qu'il ressort renforcé et enrichi de cette confrontation, ayant ajouté une couleur à sa palette de nuances.

La seconde proposition peut apparaître plus polémique, si elle est prise au premier degré : ainsi les parents prétendraient-ils, à eux seuls, être l'école? Une telle interprétation méconnaîtrait à l'évidence les travaux réalisés par l'UNAPEL depuis plus de vingt ans et notamment au cours de ses deux congrès de Lyon en 1967 et en 1998, qui avaient respectivement pour thème «La communauté éducative» et "Parents, école : un enfant à éduquer». L'affirmation même de l'existence d'une communauté éducative met à néant toute velléité de s'approprier l'école. Mais elle bouleverse définitivement un ordre établi pour rappeler ce principe appelé à devenir réalité : les parents sont dans l'école. Avant même d'y être physiquement, ils y sont par vocation nouvelle : ce sont leurs enfants qu'ils lui confient et c'est donc une part d'eux-mêmes qui, avec eux, entre dans l'institution scolaire. Sur ce point, la discussion est donc close : il n'est pas question de faire aux parents une place dans cette institution, puisqu'ils en sont l'origine, et leurs enfants, l'achèvement.

L'enjeu majeur de la relation entre parents et école consiste, en réalité, à déterminer comment parents et enseignants vont, au sein d'une institution qu'ils ont créée ensemble, se répartir les différents aspects de la mission qu'ils doivent ensemble y assumer : conduire vers son destin d'homme, en toutes ses dimensions, l'enfant pour lequel ils l'ont voulue. 
Aussi bien, c'est parce que les parents siègeront à part entière au sein du conseil d'établissement, parce qu'enseignants et parents pourront se retrouver dans des espaces de formation en commun, y compris autour de thèmes à connotation pédagogique, parce que l'association de parents d'élèves pourra remplir pleinement sa fonction de corps intermédiaire entre les parents, notamment les plus démunis en termes de capacité de dialogue et l'équipe pédagogique, que la vie de l'école cessera progressivement d'être marquée par des confrontations agressives, pour trouver son épanouissement dans un esprit de communauté éducative.

Le passage assez brutal d'une société de hiérarchies à une société de revendications, d'une conception globale de la cité à une vision individualiste du citoyen, l'affaiblissement du sens de l'existence au profit d'une interrogation angoissée sur les moyens de vivre, n’ont pas permis, voire entravé, la construction de cette communauté.

Mieux que nous, qui sommes pourtant leurs éducateurs, nos enfants ont compris que notre temps et le leur sont marqués du signe de l'échange des connaissances, des biens et des services.

Sortons du «néolithique éducatif»! Par la liberté de la pensée et de la parole, l'autonomie donnée à la vie de l'enfant, la créativité, le choix de l'honneur plutôt que celui des structures, parents et enseignants faisons ensemble le pari audacieux de l'inconnu.

Au risque de nous perdre?

\section{RELATIONS PARENTS / ÉCOLE : LA NÉCESSAIRE COÉDUCATION}

L'élève, mais au-delà de celui-ci, l'enfant, le jeune, sont au centre du système éducatif : cette donnée de base, inscrite dans la loi d'orientation sur l'éducation du 10 juillet 1989, est le socle de notre réflexion et de notre engagement sur le développement des relations parents / école.

Cette vision du jeune au cour du système éducatif traduit l'ambition humaniste, progressiste qui anime la FCPE dans le domaine de l'éducation et de la place du jeune dans notre société. Elle est à la source de la logique du partenariat qui s'incarne dans le concept de la communauté éducative, dont la réalité est garantie par la reconnaissance de la coéducation, démarche conçue et développée par la FCPE, et intégrée maintenant par l'ensemble des partenaires institutionnels.

La coéducation se développe, par sa double dimension individuelle et collective, dans une dynamique d'éducation partagée entre les familles et l'école. Elle envisage l'enfant comme un être global et recherche de façon 
permanente et continue la complémentarité et la cohérence des attitudes éducatives à son égard.

Nul ne songe, aujourd'hui, à remettre en cause, au moins dans les principes, l'importance des relations parents/école pour parvenir à la réussite de tous les jeunes. Les relations interindividuelles, les contacts qu'entretiennent les parents avec les enseignants et les personnels de l'éducation sont plus fréquents et désormais largement admis. Au plan collectif, la participation des parents d'élèves aux instances consultatives de l'Éducation nationale, des conseils d'école et d'administration jusqu'au Conseil supérieur de l'Éducation, marque une étape importante dans la reconnaissance de leur légitimité en tant que partenaires à part entière de la communauté éducative.

Toutefois, cette évolution est lente, laborieuse, car elle se heurte encore à une culture de nature essentiellement conservatrice et élitiste qui veut conserver à l'institution scolaire un statut de «chasse gardée», protégée des regards extérieurs qui pourraient être par trop critiques. En outre, la fracture sociale qui se creuse entre le monde enseignant et une partie de population constitue un facteur fort d'incompréhension.

Ainsi, de trop nombreux établissements restent encore peu accessibles, peu accueillants, et l'idée qui consiste à inviter les parents à l'école, et à inciter ceux d'entre eux qui en sont éloignés pour des raisons diverses, à y pénétrer, est encore trop peu répandue (souvenir de son propre échec, peur de s'exprimer de façon incorrecte, de ne pas comprendre, confrontation dans le seul cas où son enfant est en échec).

$\mathrm{Au}$ plan du collectif près de quinze ans après la loi d'orientation, la participation des parents d'élèves aux conseils d'école et d'administration n'est encore que tolérée. Trop souvent spectateurs et appelés à avaliser des décisions auxquelles ils n'ont pas été associés, les représentants des parents ressentent souvent leur présence comme une intrusion dans des instances qui, souvent convoquées en pleine journée, oublient que ce sont des femmes et des hommes qui, eux aussi, travaillent...

Rien, dans ce domaine, ne se réglera du jour au lendemain. Mais encore faudrait-il, au préalable, que le ministère de l'Éducation nationale fasse du bon fonctionnement de la communauté éducative un "slogan» permanent, notamment en respectant et en faisant respecter les règles et les outils qu'il a lui-même élaborés : circulaire relative à l'intervention des associations de parents d'élèves dans les établissements scolaires, fonctionnement et compétences des conseils d'école et d'administration, campagne préalable à l'élection des représentants de parents d'élèves, respect du bon déroulement de ces élections, semaine des parents à l'école.

Les parents d'élèves sont des militants de l'école publique, des militants bénévoles, des militants qui travaillent et qui donnent de leur temps, au-delà des préoccupations qui les animent pour l'avenir de leurs propres enfants, pour 
l'amélioration et le développement du service public d'éducation. Ils ont besoin de temps pour accomplir un mandat qui leur a été confié par le vote : c'est pourquoi la FCPE réclame pour eux un statut de "délégué-parent».

Enfin, si l'École ne doit pas être exposée à tous les vents, elle doit être ouverte sur la vie, accueillante et attentive à celles et à ceux qui justifient son existence et qui l'animent: les jeunes d'abord, leurs parents, les enseignants et les personnels d'éducation, du concierge au professeur en passant par l'assistante maternelle, le cuisinier, la dame de service et l'ouvrier de maintenance.

L'école a cette obligation d'ouverture, de recherche quotidienne du contact : un comportement qui ne coûte rien, à inculquer dès la formation en IUFM, qui consiste à se mettre en position de dialogue permanent avec les familles, qui sache anticiper les difficultés pour éviter de les traiter "à chaud», dans l'urgence et l'incompréhension, et qui montre, par cette attitude d'écoute, que l'école est bien là, avec la famille, pour préparer l'avenir de chaque jeune par la construction continue, progressive de ses choix de vie, autant dans son cursus professionnel que dans son rôle de citoyen. 